\title{
TCGA dataset-based construction and integrated analysis of aberrantly expressed long non-coding RNA mediated competing endogenous RNA network in gastric cancer
}

\author{
WENTING HE ${ }^{1,2^{*}}$, DACHUAN ZHANG ${ }^{3 *}$, XIAODONG LI $^{1,2}$, JUN WU $^{1}$, XUANXUAN YANG $^{1}$, \\ QI WANG ${ }^{1}$, WENBIN LU ${ }^{4}$, JINGTING JIANG $^{2}$ and CHANGPING WU ${ }^{1}$ \\ Departments of ${ }^{1}$ Oncology, ${ }^{2}$ Tumor Biological Treatment and ${ }^{3}$ Pathology, \\ The Third Affiliated Hospital of Soochow University, Changzhou, Jiangsu 213003; \\ ${ }^{4}$ Department of Medical Oncology, Wujin People's Hospital Affiliated to Jiangsu University, \\ Changzhou, Jiangsu 213000, P.R. China
}

Received May 16, 2018; Accepted September 4, 2018

DOI: $10.3892 /$ or.2018.6720

\begin{abstract}
The aberrant expression of long non-coding RNAs (lncRNAs) has been confirmed to play a pivotal role in tumor initiation and development. LncRNAs can interact with microRNAs (miRNAs) as competing endogenous RNAs (ceRNAs) to regulate the expression of target genes in various cancers. In the present study, the authors investigated the functions of lncRNAs as ceRNAs in gastric cancer (GC) and their implications for the prognosis. The RNA sequencing profiles of 372 tumor samples and 32 adjacent non-tumor gastric samples were downloaded from The Cancer Genome Atlas (TCGA) database. The differential expression of RNAs was identified using the 'edgeR' package in R language software. Survival analysis was estimated based on Kaplan-Meier curves. The Gene Ontology biological processes and the Kyoto Encyclopedia of Genes and Genomes pathways were analyzed for differentially expressed mRNAs. Finally, a total of 999 lncRNAs, 137 miRNAs and 1629 mRNAs were identified as differentially expressed (DE) in GC with log fold change (FC) thresholds $>2$ and adjusted P-values $<0.01$. A ceRNA network was constructed with 65 DElncRNAs, nine DEmiRNAs and 24 DEmRNAs. Of the 65 DElncRNAs in
\end{abstract}

Correspondence to: Dr Changping Wu, Department of Oncology, The Third Affiliated Hospital of Soochow University, 185 Juqian Street, Changzhou, Jiangsu 213003, P.R. China

E-mail: wcpijt@163.com

Dr Jingting Jiang, Department of Tumor Biological Treatment, The Third Affiliated Hospital of Soochow University, 185 Juqian Street, Changzhou, Jiangsu 213003, P.R. China

E-mail: jiangjingting@suda.edu.cn

*Contributed equally

Key words: long non-coding RNA, competing endogenous RNA, microRNA, The Cancer Genome Atlas, gastric cancer the ceRNA network, nine were identified to be significantly associated with overall survival $(\mathrm{P}<0.05)$. A total of four DElncRNAs from the ceRNA network were validated by reverse transcription-quantitative polymerase chain reaction and revealed to be associated with tumorigenesis and/or progression. In conclusion, the results of the present study provide information on the role of the ceRNA network in GC. These identified novel lncRNAs are potential candidate biomarkers and require further studies.

\section{Introduction}

Gastric cancer (GC) is one of the most common cancers and the second leading cause of cancer-associated mortality worldwide (1). There are approximately 750,000 newly diagnosed cases each year (2). However, patients with GC have a poor prognosis with a 5-year overall survival (OS) rate less than $25 \%$ (2). The OS of GC patients is considered to be associated with age, tumor node metastasis stage, histological grade and surgical approach (3). The unsatisfactory clinical outcome is greatly due to a lack of knowledge regarding the molecular pathogenesis of GC. Therefore, the identification of potential biomarkers and effective targeted therapies for GC to predict prognosis are urgently required.

Non-coding RNAs (ncRNAs) are RNA molecules that broadly exist in high eukaryotic organisms but have no ability to code proteins (4). Sana et al (5) reported that ncRNAs may play important biological roles in cellular development, chromosome formation, transcriptional regulation and RNA modification.

Long non-coding RNAs (lncRNAs), once considered transcriptional 'noise', are one subtype of ncRNA that are $>200$ nucleotides in length (6). To date, over 12,000 lncRNAs encoded in the human genome have been identified. Increasing evidence has revealed that these IncRNAs regulate proliferation, invasion and metastasis in various cancers (5,7-10). Furthermore, several studies have revealed that lncRNAs have potential in the prediction of the diagnosis and prognosis of malignant tumors including GC (11-13). 
LncRNAs play a role in crucial biological functions in multiple ways. Dysregulated lncRNAs can function as oncogenes or tumor suppressors to alter cellular pathways. LncRNAs have also been demonstrated to promote tumor cell migration and metastasis by inducing the epithelial-mesenchymal transition. Furthermore, several lncRNAs have significant effects on multidrug resistance, which is responsible for chemotherapy failure. In 2011, Salmena et al (14) proposed the competitive endogenous RNA (ceRNA) hypothesis that RNA transcripts communicate with and regulate each other by using shared miRNA response elements (MREs). This competition between mRNAs, lncRNAs and pseudogene transcripts regulates their expression using MREs to compete for binding with microRNAs (miRNAs), which is important for tumor initiation and progression $(15,16)$. Later, this hypothesis was validated experimentally by other researchers $(17,18)$.

The functions of various lncRNAs in GC have already been verified (18-21). However, there is still a lack of studies with large sample sizes and cancer-specific lncRNA biomarkers in GC. Furthermore, fewer studies have been designed to identify the potential ceRNA network.

In the present study, the authors used data from 372 tumor tissues and 32 adjacent non-tumor tissues from The Cancer Genome Atlas (TCGA), which provides information on RNA sequencing including mRNA, miRNA and lncRNA data. Then, the ceRNA network in GC was constructed. To verify the reliability of these results, four IncRNAs from the ceRNA network were randomly selected and their expression levels and functions in the GC cell line SGC-7901 were examined.

\section{Materials and methods}

Data. GC patients with RNA sequence data were enrolled in a comprehensive integrated analysis from TCGA database (www.portal.gdc.cancer.gov). The enrollment criteria were as follows: i) Gastric adenocarcinoma; ii) enough data for analysis; iii) without another type of malignant tumor and iv) naive to preoperative radiotherapy and chemotherapy. Finally, 404 samples including 372 tumor tissues and 32 adjacent non-tumor tissues were included. The study was performed in accordance with the publication guidelines provided by TCGA (www.cancergenome.nih.gov/publications/publicationguidelines).

RNA sequence data and differentially expressed analysis. The stomach adenocarcinoma (STAD) RNA expression profile data (level 3) of the corresponding patients were obtained from TCGA-STAD database (retrospect to Sep 1, 2017), which provides normalized transcriptome profiling data from high-throughput sequencing, including lncRNA, mRNA and miRNA sequencing profiles. Each sample consisted of the corresponding RNAseq, miRNAseq and clinical data. Next, the differential expression levels of lncRNAs, mRNAs and miRNAs were compared between tumor tissues and adjacent non-tumor gastric tissues. This was conducted using 'edgeR' (22), a bioconductor package based on R language (Version 3.4.3, 2017, https://www.r-project.org), to identify the differentially expressed lncRNAs (DElncRNAs) and differentially expressed mRNAs (DEmRNAs) with thresholds of $\log$ fold change (FC) $>2.0$ and the false discovery rate (FDR)-adjusted P-values $<0.01$, as well as differentially expressed miRNAs (DEmiRNAs) with thresholds of $\log$ FC $>1.5$ and FDR-adjusted P-values $<0.01$. The 'ggplot2' package in $\mathrm{R}$ was used to build the volcano plots of DElncRNAs, DEmRNAs and DEmiRNAs (23). The DEmRNAs were submitted to the Database for Annotation, Visualization, and Integrated Discovery (DAVID) (www.david.abcc.ncifcrf.gov) to be classified into different Gene Ontology (GO) and Kyoto Encyclopedia of Genes and Genomes (KEGG) annotation groups. Upregulated and downregulated genes were analyzed. The criteria were set as $\mathrm{P}<0.05$ and fold enrichment score $>1.5$.

Construction of the ceRNA network. In the present study, IncRNA-miRNA-mRNA interactions were predicted by the overlapping of the miRNA seed sequence binding site both on the chosen dysregulated lncRNAs and the significantly dysregulated mRNAs. The miRcode (Www.mircode.org) was used to predict the IncRNA-miRNA interactions (24). MiRNA-targeted mRNAs were determined by using miRDB (www.mirdb.org), Targetscan (www.targetscan.org) and miRTarBase (mirtarbase.mbc.nctu.edu.tw). To further enhance the ceRNA network reliability, the intersective lncRNAs and mRNAs were selected on the miRNA prediction and the differentially expressed data form TCGA-STAD. Those intersection lncRNAs not included in the GENCODE lncRNA annotation (www.gencodegenes.org, V27) were discarded. Cytoscape v3.5.0 software was used to construct the interactive and visual ceRNA network (25). The DEmRNA in the ceRNA network were enriched in the KEGG pathway by KOBAS 3.0 (http://kobas.cbi.pku.edu.cn) to analyze the potential functions.

Cell culture and transfection. Human GC cell line SGC-7901 and normal gastric epithelial cell line GES-1 were purchased from Shanghai Cell Bank, Chinese Academy of Sciences (Shanghai, China). Cells was cultured in Dulbecco's modified Eagle's medium (DMEM; Gibco; Thermo Fisher Scientific, Inc., Waltham, MA, USA) supplemented with $10 \%$ fetal bovine serum (FBS; Gibco; Thermo Fisher Scientific, Inc.), 100 IU/ml penicillin, and $100 \mathrm{mg} / \mathrm{ml}$ streptomycin in a humidified atmosphere at $37^{\circ} \mathrm{C}$ in an environment containing $5 \% \mathrm{CO}_{2}$. For transfection, cells were cultured up to $70 \%$ confluency and transfected with $50 \mathrm{nM}$ specific lncRNAs small interfering RNA (siRNA, si-ERVMER61, 5'-GGGUACUGUGUGUGA UAUC-3'; si-DSCR4-IT1, 5'-GAGCCAUCCAAGGAUACA A-3'; si-HULC, 5'-GGA AUUGGAGCCUUUACAA-3'; si-LINC00200, 5'-UCGCACGCUUUGCGUAGAU-3') or nontargeting siRNA (si-NC, 5'-GCAAGUAUAGCCGUAAGC A-3') (Guangzhou RiboBio Co., Ltd, Guangzhou, China), using Lipofectamine ${ }^{\circledR} 3000$ (Invitrogen; Thermo Fisher Scientific, Inc.) by incubating with OptiMem-I (Gibco; Thermo Fisher Scientific, Inc.) media for $4 \mathrm{~h}$. The cells were then cultured in fresh DMEM with $10 \%$ FBS. After $12 \mathrm{~h}$ of transfection, the relative levels of lncRNAs in transfected cells were examined using reverse transcription-quantitative polymerase chain reaction (RT-qPCR).

$R N A$ extraction and $R T-q P C R$ validation. Total RNA was extracted from cultured cells using TRIzol ${ }^{\circledR}$ LS reagent (Thermo Fisher Scientific, Inc.) according to the manufacturer's 
protocol. For lncRNA reverse transcription, cDNA was synthesized at $37^{\circ} \mathrm{C}$ for $1 \mathrm{~h}$ using 5xRT Master Mix (Toyobo Life Science, Osaka, Japan). LncRNA expression levels were quantified using qPCR with the SYBR Green I (Takara Bio, Inc., Otsu, Japan) dye detection method on ABI vii7 PCR instrument (Applied Biosystems; Thermo Fisher Scientific, Inc). The sequences of the primers used for the PCR are presented in Table I. GAPDH was used as an internal control for the expression analysis of lncRNAs. The PCR cycling conditions were as follows: $95^{\circ} \mathrm{C}$ for $5 \mathrm{~min}, 40$ cycles of $95^{\circ} \mathrm{C}$ for $10 \mathrm{sec}, 60^{\circ} \mathrm{C}$ for $20 \mathrm{sec}$, dissociation at $95^{\circ} \mathrm{C}$ for $10 \mathrm{sec}$, $60^{\circ} \mathrm{C}$ for $10 \mathrm{sec}$. Relative quantification of lncRNA expression was calculated using the $2^{-\Delta \Delta \mathrm{Cq}}$ method (26). The RT-qPCR reactions were all repeated three times.

Cell proliferation assay. The cell proliferation ability was assessed using a Cell Counting Kit-8 (CCK-8) assay (Beyotime Institute of Biotechnology, Haimen, China), and was conducted according to the manufacturer's protocol. In brief, the siRNA transfected groups and the si-NC group of SGC-7901 cells $\left(5 \times 10^{4}\right)$ were seeded into each well of a 96-well plate and cultured in $100 \mu$ I DMEM supplemented with $10 \%$ FBS for $24 \mathrm{~h}$. At $0,24,48$ and $72 \mathrm{~h}$, medium was exchanged for $10 \mu \mathrm{l} \mathrm{CCK}-8$ reagent, and the cells were incubated for $1 \mathrm{~h}$. The absorbance was measured for each well at a wavelength of $450 \mathrm{~nm}$. Cell growth was monitored every 24 h over 3 days. Each experiment was performed at least 3 times.

Transwell invasion assay. The co-culture system was used to evaluate the regulation of invasiveness in SGC-7901 cells. Cell invasion assays were performed using transwell chambers (8 $\mu \mathrm{m}$ pore size; BD Biosciences, Franklin Lakes, NJ, USA) precoated with Matrigel (Corning Life Sciences, Tewksbury, MA, USA). Approximately $1 \times 10^{6} \mathrm{GC}$ cells in serum-free DMEM media were seeded into the upper chambers following siRNA or si-NC transfection. The lower chamber contained medium with $10 \%$ FBS as a chemoattractant. After incubation for $24 \mathrm{~h}$, the non-invading cells and gel were removed from the upper chamber with cotton tipped swabs, whereas cells attached to the lower surface of the chamber were fixed and stained with crystal violet solution for $10 \mathrm{~min}$ at room temperature, after which the positive cell number was counted under an inverted microscope (IX71; Olympus Corporation, Tokyo, Japan) in five random fields. At least three chambers from three different experiments were analyzed.

Wound healing assay. Cell migration was evaluated by a wound healing assay to determine whether these lncRNAs could be involved in the regulation of the migration of GC cells. In brief, the siRNA transfected groups and the si-NC group of SGC-7901 cells were incubated in 6-well plates, respectively. A small wound area was made in the $90 \%$ confluent monolayer by using a $200 \mu \mathrm{l}$ pipette tip in a lengthwise stripe. Cells were then incubated in serum-free DMEM media at $37^{\circ} \mathrm{C}$ in a $5 \%$ $\mathrm{CO}_{2}$ incubator for $24 \mathrm{~h}$. Photographs were taken at the indicated time points using a Bx50 microscope (Olympus Corporation). A total of ten measurements were made at random intervals along the wound length. Data were averaged and expressed as a percentage of the original width. The wound healing assay was conducted in triplicate.
Table I. Primer sequences for reverse transcription-quantitative polymerase chain reaction.

\begin{tabular}{lc}
\hline Gene name & \multicolumn{1}{c}{ Primer sequences (5'-3') } \\
\hline ERVMER61 & \\
F & CAACCCACAGCAATTACACTTC \\
R & CCCAAGACTAGCCCTACAAATC \\
DSCR4-IT1 & \\
F & GAGCCATCCAAGGATACAATCA \\
R & AGTGAGCAAACACACAGAGG \\
HULC & \\
F & CATGATGGAATTGGAGCCTTTAC \\
R & CCGGCCTTTACTTCAGAGTT \\
LINC00200 & \\
F & TTCCACACACAGGACCAAAG \\
R & GCCCGATACATCAAAGCTACA \\
GAPDH & \\
F & TGACTTCAACAGCGACACCCA \\
R & CACCCTGTTGCTGTAGCCAAA
\end{tabular}

F, forward; R, reverse.

Statistical analysis. To identify the DERNAs associated with prognosis in the ceRNA network, the survival curves of differentially expressed lncRNAs, miRNAs and mRNAs were plotted using the 'survival' package in R (27). The log-rank test was used to compare significant differences between subgroups. Student's t-test was used to compare the differences between two groups. All analyses were performed using SPSS software version 24.0 for Windows (IBM SPSS, Armonk, $\mathrm{NY}$, USA). $\mathrm{P}<0.05$ was considered to indicate a statistically significant difference.

\section{Results}

Patient characteristics. The detailed clinicopathological characteristics of enrolled patients are listed in Table II. All 372 patients were pathologically diagnosed with gastric adenocarcinoma. The median age of the patients was 67 (ranging from 35 to 90). There were 239 (64.2\%) males and $133(35.8 \%)$ females. The majority of patients were Caucasian (63.7\%) and without distant metastasis $(87.9 \%)$.

DEmRNAs and DEmiRNAs in GC. The present study identified the significant DEmRNAs and DEmiRNAs in 372 tumor samples compared with the 32 adjacent non-tumor samples from the TCGA database. With $\log F C>2$ and adjusted P-value $<0.01$, a total of 1629 DEmRNAs were identified by the 'edgeR' package in R, including 856 (52.5\%) upregulated and 773 (47.5\%) downregulated genes. Furthermore, 137 miRNAs that were differentially expressed from the TCGA database were identified with $\log \mathrm{FC}>1.5$, adjusted P-value $<0.01$, of which $111(81.0 \%)$ were upregulated and 26 (23.4\%) were downregulated. The volcano plots of DEmRNAs and DEmiRNAs were built using the 'ggplot2' package in R (Fig. 1A and B). 
Table II. Clinicopathological characteristics of 372 patients with gastric cancer.

\begin{tabular}{|c|c|c|}
\hline Parameter & Patients & $(\%)$ \\
\hline \multicolumn{3}{|l|}{ Age (years) } \\
\hline$\leq 60$ & 123 & 33.1 \\
\hline$>60$ & 249 & 66.9 \\
\hline \multicolumn{3}{|l|}{ Gender } \\
\hline Male & 239 & 64.2 \\
\hline Female & 133 & 35.8 \\
\hline \multicolumn{3}{|l|}{ Race } \\
\hline Asian & 73 & 19.6 \\
\hline White & 237 & 63.7 \\
\hline Black or African American & 11 & 3.0 \\
\hline Not available & 51 & 13.7 \\
\hline \multicolumn{3}{|l|}{ Histologic grade } \\
\hline High (G1-2) & 145 & 39.0 \\
\hline Low (G3) & 218 & 58.6 \\
\hline $\mathrm{GX}^{\mathrm{a}}$ & 9 & 2.4 \\
\hline \multicolumn{3}{|l|}{ Histologic subtype } \\
\hline Signet ring type & 10 & 2.7 \\
\hline Diffuse type & 63 & 16.9 \\
\hline Not otherwise specified & 206 & 55.4 \\
\hline Mucinous type & 19 & 5.1 \\
\hline Papillary type & 5 & 1.3 \\
\hline Tubular type & 68 & 18.3 \\
\hline Not available & 1 & 0.3 \\
\hline \multicolumn{3}{|l|}{ Pathologic stage } \\
\hline Stage I & 52 & 14.0 \\
\hline Stage II & 110 & 29.6 \\
\hline Stage III & 152 & 40.9 \\
\hline Stage IV & 37 & 9.9 \\
\hline Not available & 21 & 5.6 \\
\hline \multicolumn{3}{|l|}{ Pathologic T } \\
\hline $\mathrm{T} 1$ & 19 & 5.1 \\
\hline $\mathrm{T} 2$ & 79 & 21.2 \\
\hline $\mathrm{T} 3$ & 166 & 44.6 \\
\hline $\mathrm{T} 4$ & 100 & 26.9 \\
\hline $\mathrm{TX}^{\mathrm{b}}$ & 8 & 2.2 \\
\hline \multicolumn{3}{|l|}{ Pathologic N } \\
\hline NO & 110 & 29.6 \\
\hline N1 & 96 & 25.8 \\
\hline N2 & 76 & 20.4 \\
\hline N3 & 73 & 19.6 \\
\hline $\mathrm{NX}^{\mathrm{c}}$ & 17 & 4.6 \\
\hline \multicolumn{3}{|l|}{ Pathologic M } \\
\hline M0 & 327 & 87.9 \\
\hline M1 & 25 & 6.7 \\
\hline $\mathrm{MX}^{\mathrm{d}}$ & 20 & 5.4 \\
\hline \multicolumn{3}{|l|}{ Status } \\
\hline Death & 149 & 40.1 \\
\hline Alive & 222 & 59.7 \\
\hline Not available & 1 & 0.3 \\
\hline
\end{tabular}

${ }^{a}$ Histologic grade unknown; bdepth of tumor invasion unknown; 'regional lymph node unknown; ${ }^{\mathrm{d}}$ metastasis status unknown.
Table III. miRNAs that may target gastric cancer specific mRNAs.

\begin{tabular}{ll}
\hline miRNAs & \multicolumn{1}{c}{ mRNAs } \\
\hline hsa-mir-96 & TRIB3 \\
hsa-mir-143 & COL1A1, SERPINE1 \\
hsa-mir-145 & MEST, SERPINE1 \\
hsa-mir-195 & ALOX12, BTG2, CBX2, CCNE1, CEP55, \\
& CLSPN,E2F7, HOXA10, KIF23, TMEM100, \\
& TPM2 \\
hsa-mir-204 & CHRDL1, HOXC8, IL11, NPTX1 \\
hsa-mir-205 & ESRRG \\
hsa-mir-372 & ATAD2, CADM2, LEFTY1, TMEM100 \\
hsa-mir-373 & ATAD2, CADM2, LEFTY1, TMEM100 \\
hsa-mir-519d & ATAD2, FAM129A, KIF23 \\
\hline
\end{tabular}

The volcano plot of DElncRNAs was built using the same method (Fig. 1C).

To further validate the potential functional implication of 1629 DEmRNAs, functional enrichment analysis of DEmRNA was performed based on the GO and KEGG pathways (P-value $<0.05$ and fold enrichment score $>1.5$ ). It was demonstrated that upregulated DEmRNAs were significantly enriched in $165 \mathrm{GO}$ terms, with 115 in biological process (BP), 17 in cellular component (CC) and 33 in molecular function (MF). The downregulated DEmRNAs were significantly enriched in $220 \mathrm{GO}$ terms, with 123 in BP, 38 in CC and 59 in MF. Fig. 2 shows the top 20 enriched GO terms for DEmRNAs based on the P-values. KEGG pathway analysis indicated that 12 pathways corresponded to upregulated DEmRNAs and 43 pathways corresponded to downregulated DEmRNAs. 'Neuroactive ligand-receptor interaction' and 'Metabolism of xenobiotics by cytochrome P450' were the most enriched pathways in the upregulation and downregulation groups, respectively (Fig. 3). Furthermore, among these pathways, chemical carcinogenesis, transcriptional misregulation in cancer, the chemokine signaling pathway and signaling pathway regulation of stem cells were considered cancer-associated pathways.

Next, 137 DEmiRnAs that targeted mRNAs were predicted using miRDB, Targetscan and miRTarBase, and a total of 925 mRNAs were included in all the three databases. Finally, 24 mRNAs were selected that interacted with 9 DEmiRNAs (Table III) by intersecting 925 targeted mRNAs and 1629 DEmRNAs. These 24 mRNAs were further used to construct the ceRNA network.

DElncRNAs in GC. According to the cut-off criteria of $\log \mathrm{FC}>2$ and adjusted P-value $<0.01$, the present study then identified 999 DElncRNAs between GC tumor tissues and adjacent non-tumor tissues from TCGA database, of which 791 were upregulated and 208 were downregulated. The volcano plot of DElncRNAs is presented in Fig. 1C. The 9 key DEmiRNAs mentioned in Table III were then used to predict the corresponding IncRNAs using the miRcode 
A

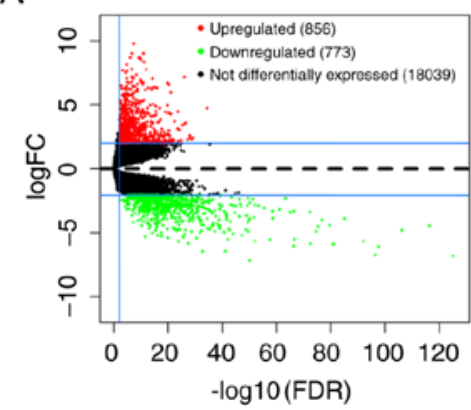

B

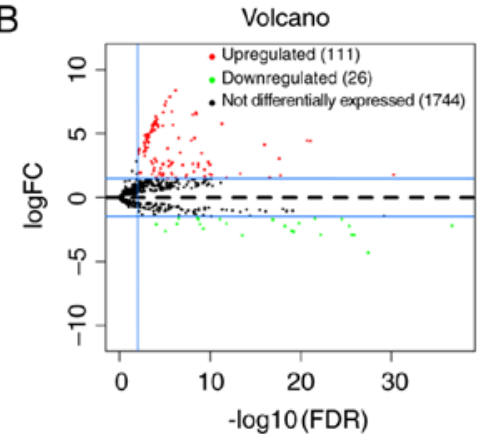

C

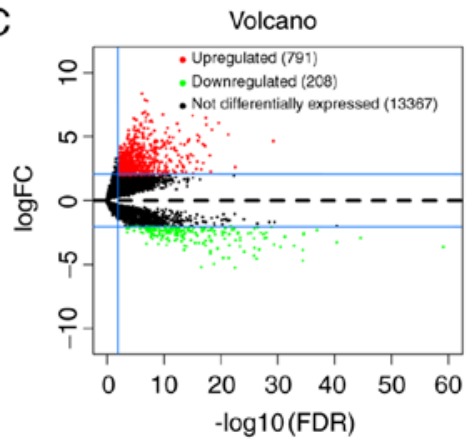

Figure 1. Volcano plots were used to visualize and assess the variation of (A) mRNAs, (B) microRNAs, and (C) long non-coding RNAs expression between gastric cancer tissues and adjacent non-tumor gastric tissues. The values of the $\mathrm{x}$ - and $\mathrm{y}$-axes indicate the averaged normalized signal values of the group (log scaled).
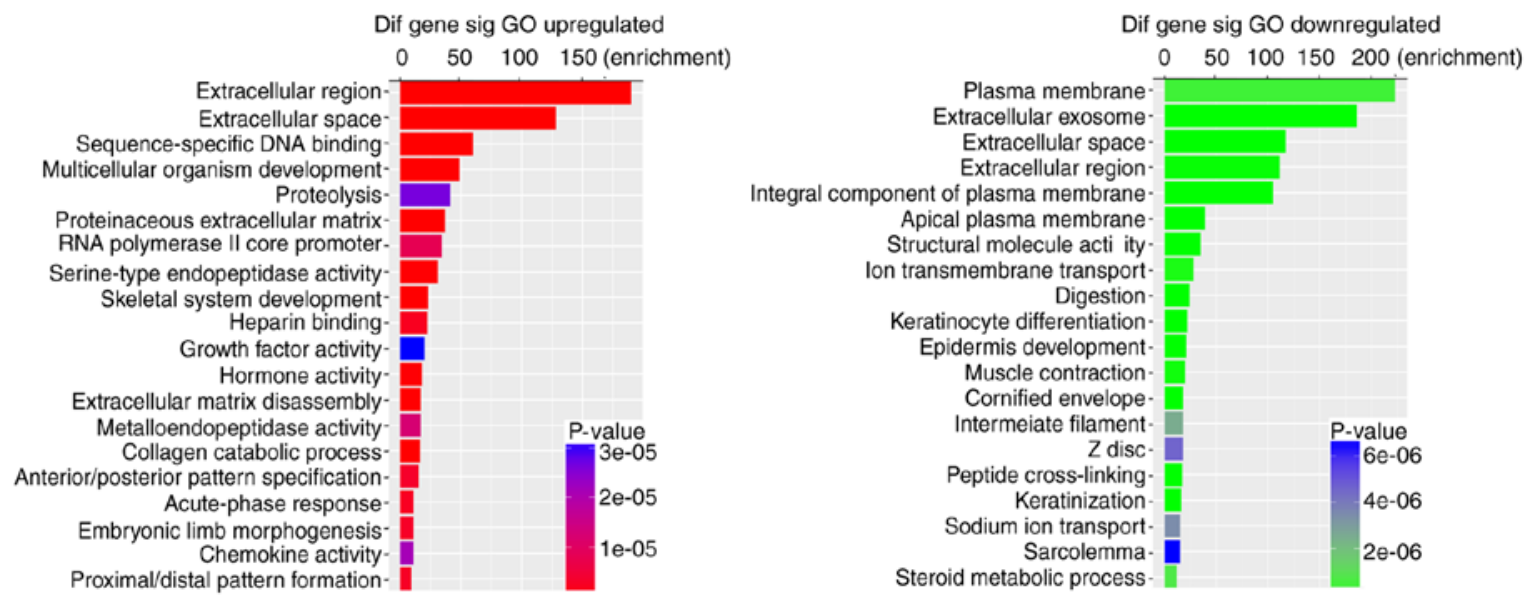

Figure 2. Top 20 enriched GO terms for differentially expressed mRNAs (the bar plot shows the enrichment scores of the significantly enriched GO terms). GO, Gene Ontology.

database. It was revealed that 9 DEmiRNAs interacted with the 63 DElncRNAs retrieved in the miRcode database (Table IV). Moreover, the selected 63 DElncRNAs (42 upregulated, 21 downregulated) were all identified in the GENCODE IncRNA annotation (V27), and all of them were used to build the ceRNA network.

Construction of a ceRNA network. To better understand the mechanism by which lncRNA mediates mRNA through combining with miRNA in GC, the present study constructed a lncRNA-miRNA-mRNA ceRNA network using Cytoscape 3.5.0 according to the information provided in Tables III and IV. The network is presented in Fig. 4. In total, 63 DElncRNAs, 9 DEmiRNAs and 24 DEmRNAs were involved in the network. Certain mRNAs involved in the ceRNA network have been reported to be cancer-associated genes, including Tribbles Pseudokinase 3, Serpin Family E Member 1 (SERPINE1), Mesoderm Specific Transcript, BTG Anti-Proliferation Factor 2, Chromobox 2, Cyclin E1 (CCNE1), Centrosomal Protein 55 (CEP55), E2F Transcription Factor 7, Homeobox (HOX)A10, Kinesin Family Member 23, Transmembrane Protein 100, HOXC8, interleukin 11 and ATPase Family, AAA Domain Containing 2 (ATAD2). Subsequently, a Kaplan-Meier curve was used to analyze the association between the differentially expressed genes in the
ceRNA network and the OS of patients with GC. As a result, 9 out of 63 DElncRNAs were all significantly negatively associated with OS ( $\mathrm{P}<0.05$; Fig. 5). Additionally, 6 out of 24 DEmRNAs were considered to be significantly associated with OS. Increased levels of CEP55, Claspin (CLSPN) and HOXA10 were associated with longer survival time, while higher levels of Chordin Like 1 (CHRDL1), Neuronal Pentraxin 1 and SERPINE1 were associated with poorer prognosis ( $\mathrm{P}<0.05$; Fig. 6). However, no association was observed between the key 9 DEmiRNAs and OS. Finally, the present study also analyzed the 24 DEmRNAs involved in the ceRNA network to understand the signaling pathways indirectly regulated by lncRNAs by KOBAS 3.0 (http://kobas.cbi.pku. edu.cn). As presented in Table V, 4 KEGG pathways with statistical significance were identified. A total of three of the pathways, including the p53 signaling pathway, microRNAs in cancer and the Phosphatidylinositol 3-kinase (PI3K)- RAC-a serine/threonine-protein kinase (Akt) signaling pathway, were involved in the pathogenesis of GC. One gene (CCNE1) was involved in more than one KEGG pathway in accordance with GC development.

$R T$ - $q P C R$ validation of key lncRNAs. To confirm the credibility of the bioinformatics results and the validity of the aforementioned analyzed results, the present study randomly selected 

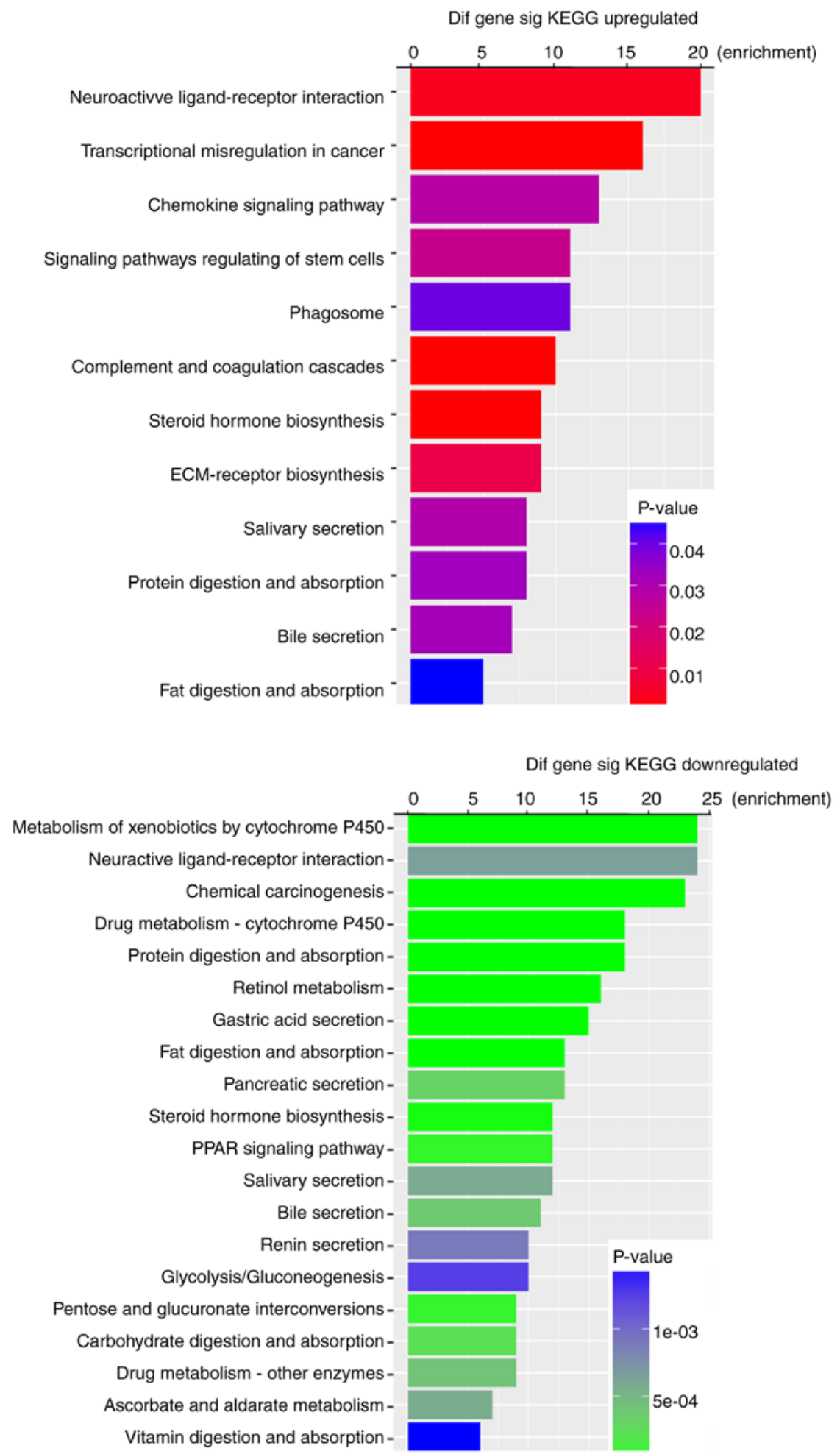

Figure 3. Top 20 enriched KEGG pathways for differentially expressed mRNAs (the bar plot shows the enrichment scores of the signifcantly enriched KEGG pathways). KEGG, Kyoto Encyclopedia of Genes and Genomes.

4 upregulated key lncRNAs (ERVMER61, DSCR4-IT1, HULC and LINC00200) from the ceRNA network and examined the expression levels of these 4 lncRNAs between the SGC-7901 cell line and the human gastric epithelial mucosa cell line GES-1 by RT-qPCR. As presented in Fig. 7, the results revealed that the expression levels of these 4 lncRNAs were all significantly increased in the SGC-7901 cell line relative to the human gastric epithelial mucosa cell line GES-1 $(\mathrm{P}<0.05)$.
The conclusions were consistent with TCGA database and bioinformatics predicted results.

Exploration of the biological functions of key lncRNAs. To better understand the biological functions of the four key lncRNAs, the present study further investigated their impact on tumor proliferation, invasion and migration in the SGC7901 cell line. The 4 key lncRNAs (ERVMER61, DSCR4-IT1, HULC 
Table IV. miRNAs that may target gastric cancer specific lncRNAs.

\begin{tabular}{|c|c|}
\hline miRNAs & lncRNAs \\
\hline hsa-mir-96 & $\begin{array}{l}\text { ADAMTS9-AS1, ADAMTS9-AS2, C8orf31, DLEU7-AS1, ERVMER61-1, } \\
\text { FRMD6-AS2, HCG22, LINC00114, LINC00221, LINC00534, LRRC3-AS1, } \\
\text { MYB-AS1, NKX2-1-AS1, RBMS3-AS3, UCA1 }\end{array}$ \\
\hline hsa-mir-143 & $\begin{array}{l}\text { AC074035.1, AC110491.1, ADAMTS9-AS2, AL139002.1, AL391152.1, } \\
\text { ARHGEF26-AS1, C15orf54, C17orf77, C2orf48, CECR3, FRMD6-AS2, } \\
\text { HOTAIR, HOTTIP, LINC00114, LINC00163, LINC00221, LINC00200, } \\
\text { LINC00284, LINC00460, LINC00524, MIR205HG, PART1, UCA1 }\end{array}$ \\
\hline hsa-mir-145 & $\begin{array}{l}\text { ADAMTS9-AS1, ADAMTS9-AS2, AP003027.1, DLX6-AS1, HCG22, } \\
\text { LINC00052, LINC00184, LINC00330, MIR205HG, NKX2-1-AS1, PART1 }\end{array}$ \\
\hline hsa-mir-195 & $\begin{array}{l}\text { ABCA9-AS1, AC034229.1, AC087269.1, AC092422.1, AP002478.1, } \\
\text { AP003027.1, C15orf54, C2orf48, CECR3, DLEU7-AS1, DLX6-AS1, HCG22, } \\
\text { HOTTIP, IL20RB-AS1, LINC00200, LINC00284, LINC00326, LINC00355, LINC00330, PART1 }\end{array}$ \\
\hline hsa-mir-204 & $\begin{array}{l}\text { AC006449.1, AC034229.1, AC110491.1, ADAMTS9-AS2, ANO1-AS2, ARHGEF26-AS1, C17orf77, } \\
\text { C2orf48, C8orf31, DLX6-AS1, DSCR4-IT1, ERVMER61-1, FRMD6-AS2, HOTAIR, HOTTIP, HULC, } \\
\text { LINC00114, LINC00221, LINC00200, LINC00330, LINC00332, LINC00410, LINC00501, } \\
\text { LINC00524, LRRC3-AS1, NKX2-1-AS1, MIR205HG, PART1, RBMS3-AS3, VCAN-AS1 }\end{array}$ \\
\hline hsa-mir-205 & $\begin{array}{l}\text { AC006449.1, AC110491.1, ADAMTS9-AS2, AP002478.1, ARHGEF26-AS1, C20orf166-AS1, } \\
\text { ERVMER61-1, HOTTIP, IL20RB-AS1, LINC00184, LINC00284, LINC00326, LINC00330, } \\
\text { LINC00410, LINC00524, LINC00534, MIR205HG, PART1 }\end{array}$ \\
\hline hsa-mir-372 & $\begin{array}{l}\text { AC011374.1, AC061975.6, ADAMTS9-AS2, ANO1-AS2, AP002478.1, ARHGEF26-AS1, C15orf54, } \\
\text { C17orf77, C20orf166-AS1, C2orf48, C8orf31, CECR3, DLX6-AS1, HOTTIP, LINC00184, LINC00221, } \\
\text { LINC00330, LINC00393, LINC00534, VCAN-AS1 }\end{array}$ \\
\hline hsa-mir-373 & $\begin{array}{l}\text { AC011374.1, AC061975.6, ADAMTS9-AS2, ANO1-AS2, AP002478.1, ARHGEF26-AS1, C15orf54, } \\
\text { C17orf77, C20orf166-AS1, C2orf48, C8orf31, CECR3, DLX6-AS1, HOTTIP, LINC00184, LINC00221, } \\
\text { LINC00330, LINC00393, LINC00534, VCAN-AS1 }\end{array}$ \\
\hline hsa-mir-519d & $\begin{array}{l}\text { AC061975.6, AC092422.1, AL391152.1, ANO1-AS2, AP002478.1, AP003027.1, ARHGEF26-AS1, } \\
\text { C17orf77, C20orf166-AS1, C2orf48, DLX6-AS1, H19, HOTAIR, HOTTIP, IGF2-AS, LINC00052, } \\
\text { LINC00184, LINC00200, LINC00221, LINC00284, LINC00330, LINC00365, LINC00410, } \\
\text { LINC00454, NKX2-1-AS1, TDRG1, VCAN-AS1 }\end{array}$ \\
\hline
\end{tabular}

Table V. Kyoto Encyclopedia of Genes and Genomes pathways enriched by the coding genes involved in competing endogenous RNA network.

\begin{tabular}{llll}
\hline Pathways ID & \multicolumn{1}{c}{ Description } & P-value & Genes \\
\hline hsa04115 & p53 signaling pathway & 0.002088 & CCNE1, SERPINE1 \\
hsa04933 & AGE-RAGE signaling pathway in diabetic complications & 0.004497 & COL1A1, SERPINE1 \\
hsa05206 & MicroRNAs in cancer & 0.028122 & CCNE1, KIF23 \\
hsa04151 & PI3K-Akt signaling pathway & 0.042544 & CCNE1, COL1A1 \\
\hline
\end{tabular}

and LINC00200) were knocked down in SGC-7901 cells via transfection with corresponding siRNAs. The expression levels of the four lncRNAs in SGC-7901 cells were evidently downregulated following siRNA transfection (Fig. 8A). Growth curves generated from CCK8 proliferation assays demonstrated that knockdown of DSCR4-IT1 and LINC00200 significantly inhibited cell proliferation in SGC-7901 cells at both 48 and $72 \mathrm{~h}(\mathrm{P}<0.05)$, whereas knockdown of ERVMER61 and HULC only resulted in significant inhibition of the growth ability of SGC-7901 cells at 72 and 48 h, respectively (Fig. 8B). These findings indicated that DSCR4-IT1 and LINC00200 may behave as oncogenes to promote GC cell proliferation. In addition, reduced cell invasion in SGC-7901 cells was observed after si-ERVMER61, si-DSCR4-IT1, si-HULC and si-LINC00200 transfection (Fig. 8C and D). Furthermore, a decrease in cell migration was also observed when HULC 


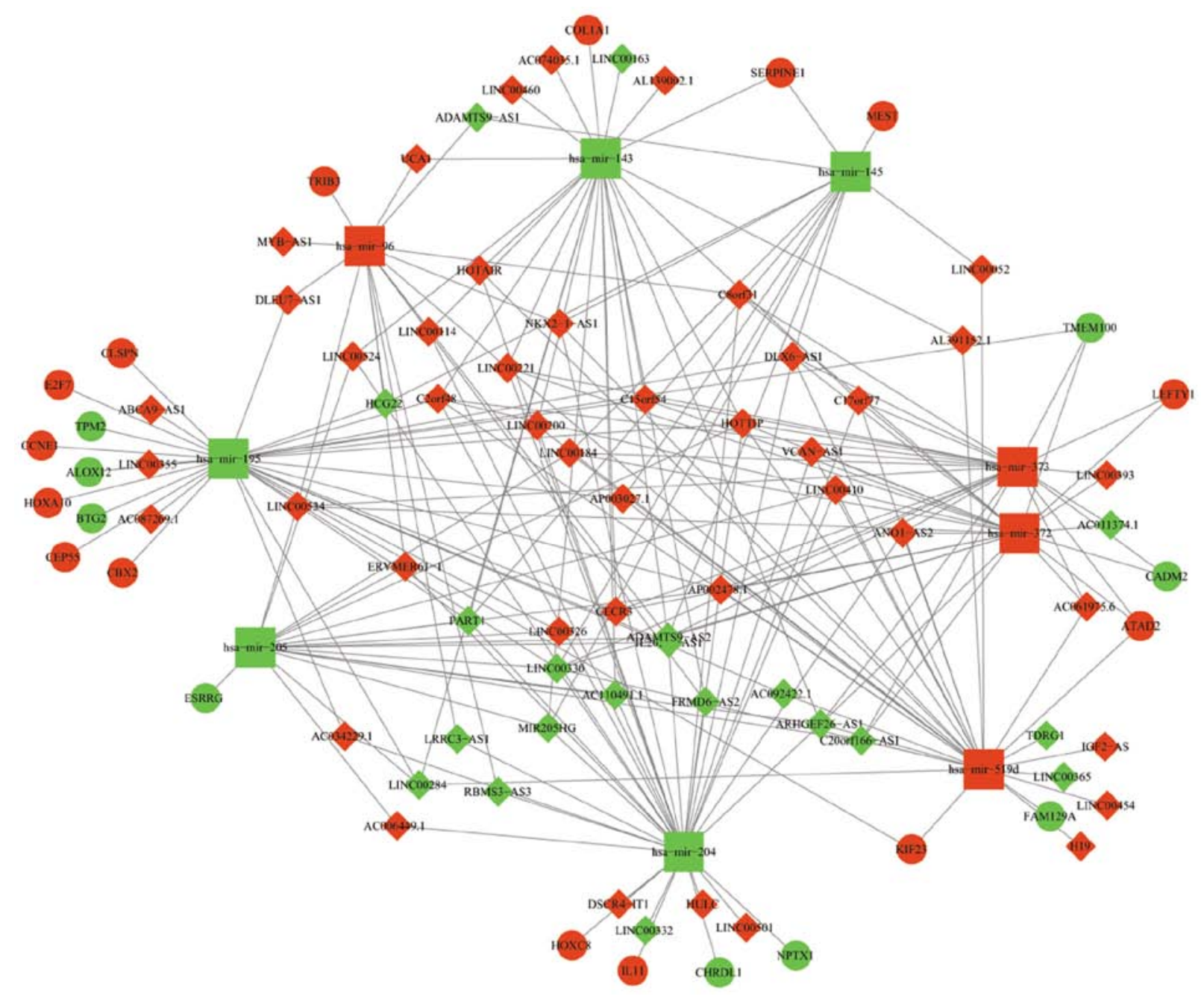

Figure 4. The lncRNA-miRNA-mRNA ceRNA network. Green squares, downregulated miRNAs; green circles, downregulated mRNAs; green diamonds, downregulated lncRNAs. Red squares, upregulated miRNAs; red circles, upregulated mRNAs; red diamonds, upregulated lncRNAs; miRNAs, microRNAs; lncRNAs, long non-coding RNAs.

or LINC00200 were significantly downregulated in a wound healing assay (Fig. 8E and F), suggesting that HULC and LINC00200 can promote GC cell migration in vitro.

\section{Discussion}

Previously, multiple studies have suggested that lncRNAs play a crucial role in the modulation of tumor behavior through various complex mechanisms such as epigenetic regulation, transcriptional regulation, and post-transcriptional regulation (28-30). Until now, however, there have been few studies of the expression profiles of IncRNAs in GC, and a few of them were reported through a microarray or sequencing with a small sample size (31). There is a complex regulatory network association between lncRNAs and miRNAs or lncRNAs and mRNAs in GC, and these networks play important roles in the pathogenesis and progression of GC $(32,33)$. However, limited data are available. In the present study, the authors aimed to investigate the interactions among lncRNAs, miRNAs and mRNAs by constructing a ceRNA network and searched for lncRNAs that may be promising biomarkers for GC.

In the present study, 1629 mRNAs were differentially expressed from $372 \mathrm{GC}$ tumor tissues and 32 non-tumor gastric tissues based on the RNA sequence data from TCGA. With bioinformatic technologies, 24 DEmRNAs were selected to construct the ceRNA network. Then, the enrichment of functions and signaling pathways of the DEmRNAs were analyzed using GO and KEGG, respectively. The GO results indicated the functions of significant differences mostly in terms of the biological process, cellular component and molecular function. Among the KEGG pathway analysis results, some were considered to be cancer associated, such as transcriptional misregulation in cancer, signaling pathway regulation of stem cells, p53 signaling pathway, PI3K-Akt signaling pathways, and microRNAs in cancer. Various previous studies have revealed that the PI3K-Akt and p53 signaling pathways play an important role in cell proliferation and cell apoptosis reduction (34-36). It has also been reported that the lncRNAs AK023391 and MEG3 impact GC cell function via the PI3K-Akt or p53 signaling pathway $(37,38)$. Furthermore, three DEmRNAs (SERPINE1, CCNE1 and COL1A1) from the ceRNA network involved in the PI3K-Akt or p53 signaling pathway were revealed to play a crucial role in the pathogenesis, prognosis and molecular therapy of GC (39-41). In addition, other cancer-associated pathways identified in the study, including miRNAs in 

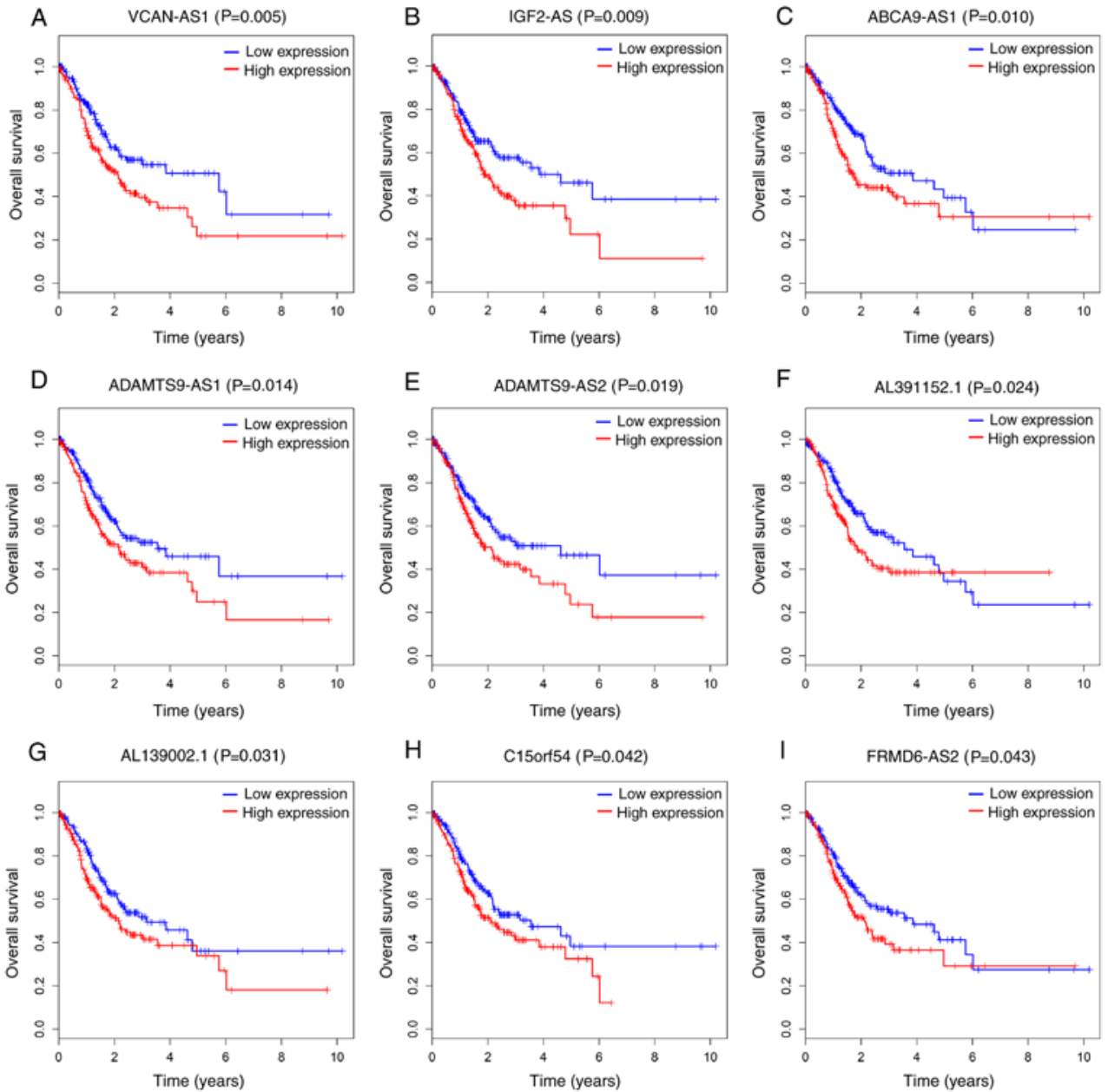

Figure 5. Kaplan-Meier survival curves for 9 long non-coding RNAs associated with overall survival. Horizontal axis: overall survival time (years); vertical axis: survival function. Nine DElncRNAs are presented (P<0.05), including (A) VCAN-AS1, (B) IGF2-AS, (C) ABCA9-AS1, (D) ADAMTS9-AS1, (E) ADAMTS9-AS2, (F) AL391152.1, (G) AL139002.1, (H) C15orf54 and (I) FRMD6-AS2.
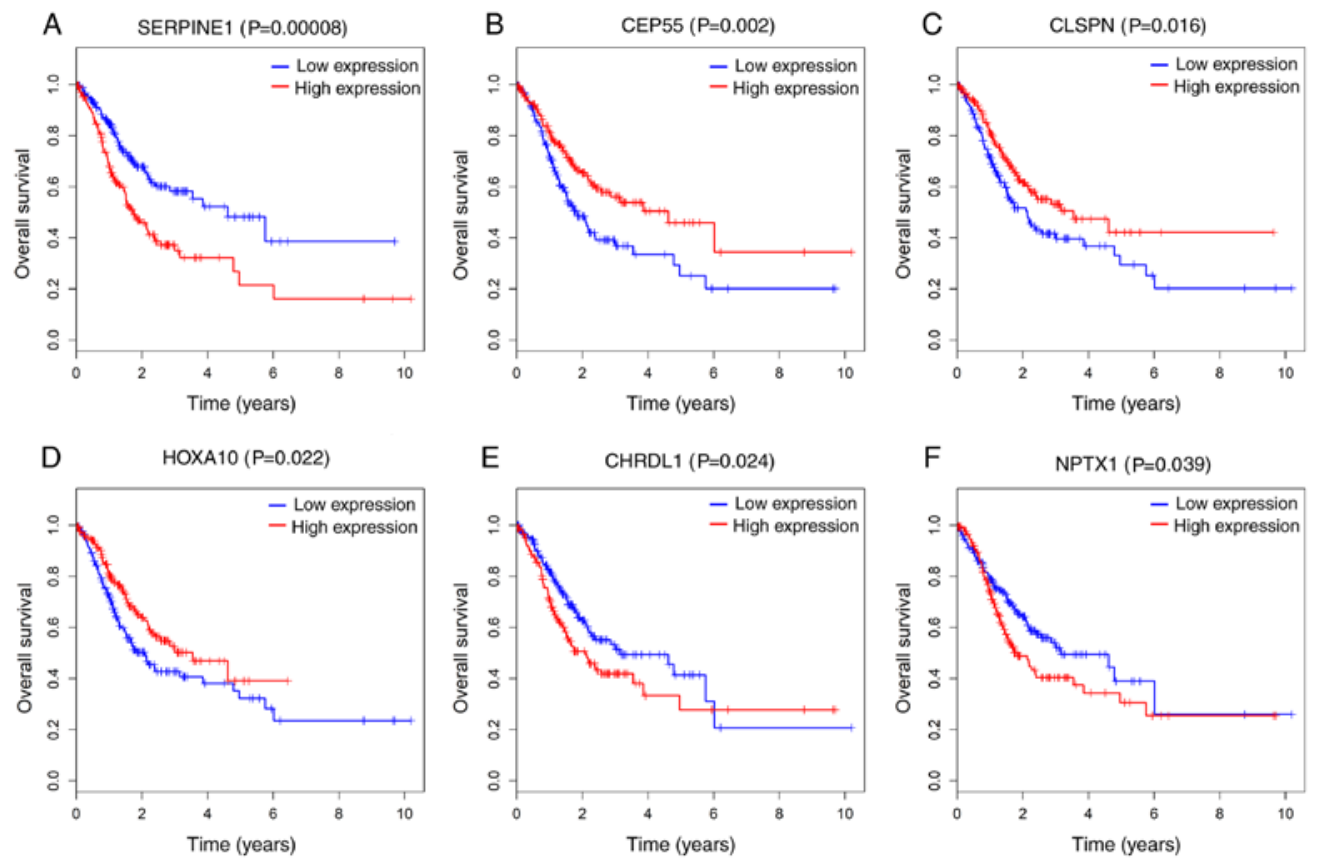

Figure 6. Kaplan-Meier survival curves for 6 protein-coding genes associated with overall survival. Horizontal axis: overall survival time (years); vertical axis: Survival function. Six DEmRNAs are presented (P<0.05), including (A) SERPINE1, (B) CEP55, (C) CLSPN, (D) HOXA10, (E) CHRDL1 and (F) NPTX1. SERPINE 1, Serpin Family E Member 1; CEP55, Centrosomal Protein 55; CLSPN, Claspin; HOXA10, Homeobox A10; CHRDL1, Chordin Like 1; NPTX1, Neuronal Pentraxin 1. 


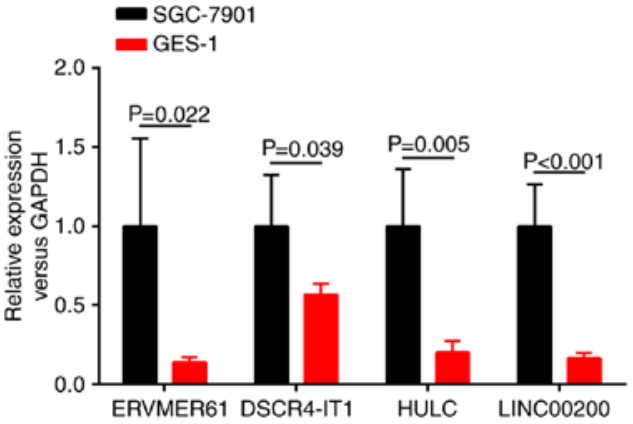

Figure 7. Reverse transcription-quantitative polymerase chain reaction validation of 4 differentially expressed key lncRNAs. Relative expression levels of 4 lncRNAs in SGC-7901 cell line compared with GES-1 cell line. lncRNAs, long non-coding RNAs. cancer, transcriptional misregulation in cancer and chemical carcinogenesis, were also reported in previous studies $(21,42)$, indicating that the present results are highly reliable.

The ceRNA hypothesis has been proposed as a novel regulatory mechanism between non-coding RNA and coding RNA (14). LncRNA can regulate gene expression by interacting with the miRNAs by MREs. For example, Jin et al (43) revealed that the IncRNA SNHG15 contributes to non-small cell lung cancer (NSCLC) tumorigenesis by regulating the CDK14 protein via sponging miR-486, providing novel insight into NSCLC pathogenesis and a potential therapeutic strategy for NSCLC patients. Yu et al (44) demonstrated that lncRNA PVT1 can promote the metastasis and proliferation of colon cancer by suppressing the miR-30d-5p/Runt Related

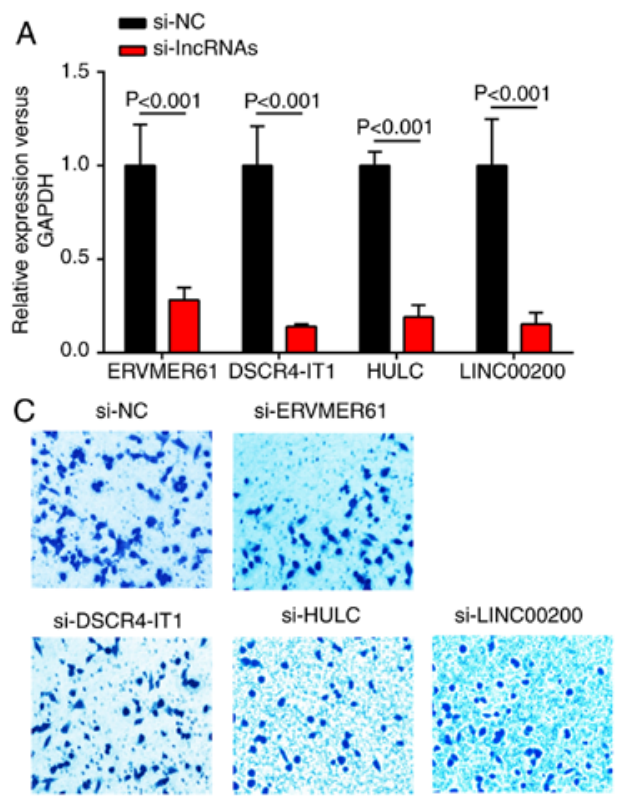

B
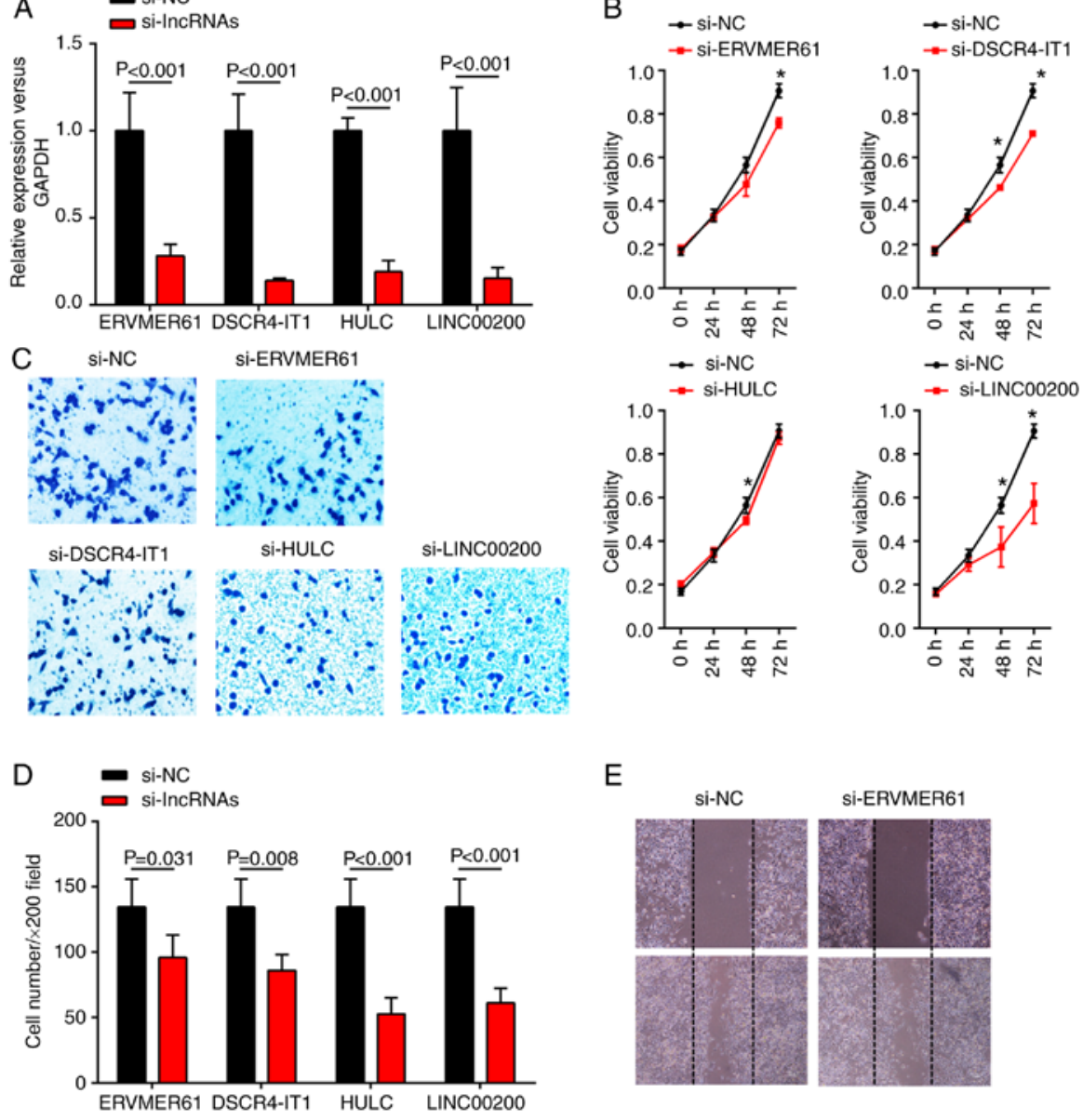

E
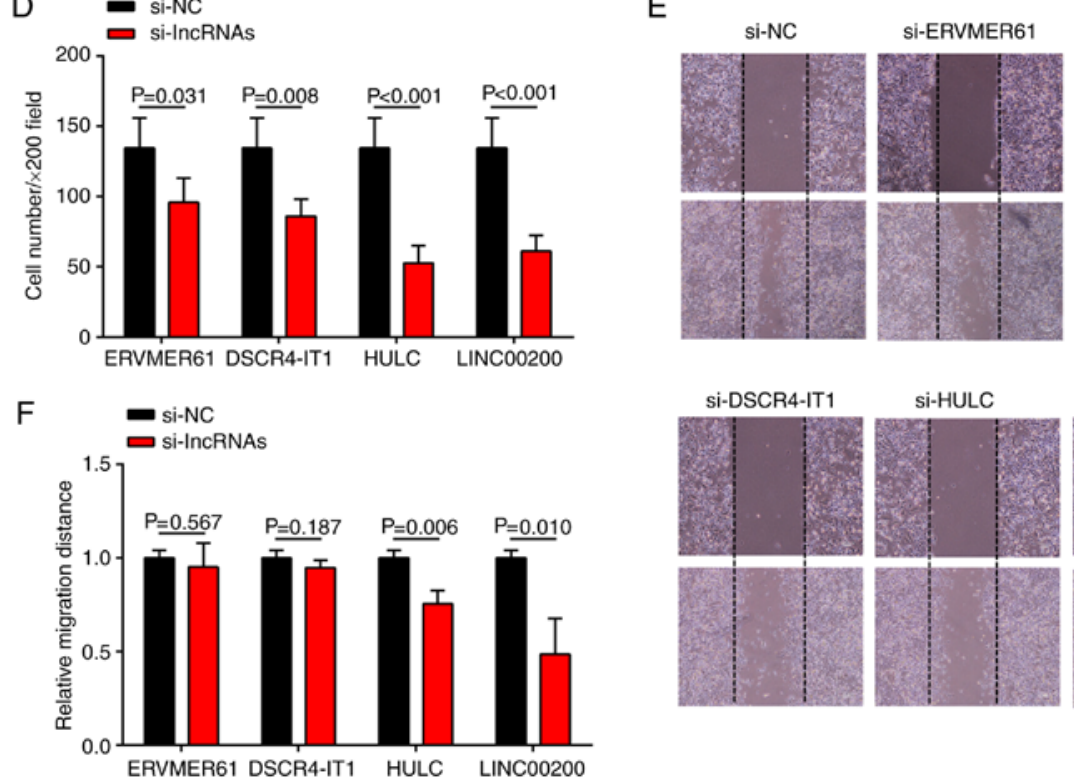

si-LINC00200
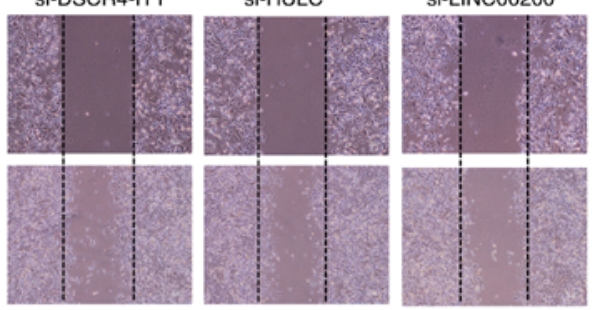

Figure 8. Effects of IncRNAs on the biological behaviors of SGC-7901 cells. (A) Knockdown of the different lncRNAs in SGC-7901 cells were confirmed by reverse transcription-quantitative polymerase chain reaction. (B) Cell proliferation measured by Cell Counting Kit-8 assays in SGC-7901 cells transfected with siRNAs or si-NC. ${ }^{*} \mathrm{P}<0.05$. (C and D) Transwell invasion assays revealed that the number of invaded cells was significantly decreased in the 4 siRNA transfected groups than in the si-NC transfected group $(\mathrm{P}<0.05)$. (E and F) Wound healing assays revealed that the migration distance of cells was significantly decreased in the 2 siRNA transfected groups (si-HULC, si-LINC00200) than in the si-NC transfected group $(\mathrm{P}<0.05)$. lncRNAs, long non-coding RNAs; si, small interfering; NC, negative control. 
Transcription Factor 2 axis. To understand the internal contact between IncRNA-miRNA-mRNA in the development and progression of GC, the present study constructed the ceRNA network by bioinformatics prediction based on differences in lncRNA, miRNA and mRNA expression from TCGA. Certain lncRNAs that exist in the network have been proven to interact with miRNA and mRNA and may be potential biomarkers in the diagnosis, therapy and prognosis of GC, including UCA1, HOTTIP, HOTAIR and H19 (45-48). Additionally, a few genes from the ceRNA network, including CHRDL1, COL1A1, HOXC8 and ATAD2, have been reported to act as tumor oncogenes or suppressor genes, participating in tumor growth, invasion and metastasis $(41,49-51)$.

Then, the present study analyzed the association between the 63 key lncRNAs and OS. The results indicated that 9 of them were significantly associated with survival and could be considered as potential prognostic markers for GC. Among the 9 lncRNAs, high IGF2-AS expression has been reported to be associated with poor clinical outcomes in ovarian cancer (52), and ADAMTS9-AS2 has also been identified as a poor prognostic biomarker of colorectal cancer (53) and glioma (54) with DNA methyltransferase-1. However, the roles of remaining 7 lncRNAs (ABCA9-AS1, ADAMTS9-AS1, AL139002.1, AL391152.1, C15orf54, FRMD6-AS2 and VCAN-AS1) have not yet been reported.

Finally, four key lncRNAs (ERVMER61, DSCR4-IT1, HULC, LINC00200) were randomly selected from the network and their expression levels analyzed in the SGC-7901 and GEC-1 cell lines. The expression data from TCGA and the verification results of the GC cell line were in accordance. The present study also explored the functions of the 4 lncRNAs in the SGC-7901 cell line and tried to search for biomarkers that may affect the biological behaviors of GC cells. The results demonstrated that all 4 lncRNAs were associated with tumor invasion, and parts of them were associated with tumor proliferation and/or migration. It was worth noting that LINC00200 played an important role in the tumor proliferation and progression of GC, which provides us with a novel biomarker and perhaps a potential target for GC.

There are still some limitations to the present study. First, the present study validated the results of TCGA in a GC cell line in vitro. Thus, an in vivo experiment using patient $\mathrm{GC}$ samples is required to further confirm the results. Second, other GC cell lines with different differentiations are needed to explore and verify the functions of key lncRNAs.

In conclusion, the results identified the cancer-specific mRNAs and noncoding RNAs in GC by bioinformatics analysis of large-scale samples from TCGA database. The study therefore provides insights into the ceRNA regulatory network in GC and describes lncRNAs that are associated with GC as diagnostic, therapeutic and prognostic biomarkers.

\section{Acknowledgements}

Not applicable.

\section{Funding}

The present study was supported by grants from the National Natural Science Foundation of China (grant nos. 31570877 and 31570908), Changzhou Health and Family Planning Commission Youth Talent Science and Technology Project (grant no. QN201709), Changzhou High-Level Medical Talents Training Project (grant no. 2016CZBJ054), Changzhou Health and Family Planning Commission Program (grant no. ZD201608) and Wujin Sci and Tech Program (grant no. WS201606).

\section{Availability of data and materials}

The datasets used during the present study are available from the corresponding author upon reasonable request.

\section{Authors' contributions}

WH conceived, designed and performed the study. DZ, XL, JW, XY, QW, WL, JJ and CW were also involved in the conception of the study and gave their advice in the process of the research. DZ assisted WH with the data analysis. WH wrote the paper. WH and DZ reviewed and edited the manuscript. All authors have read and approved the manuscript and agree to be accountable for all aspects of the research in ensuring that the accuracy or integrity of any part of the work are appropriately investigated and resolved.

\section{Ethics approval and consent to participate}

Not applicable.

\section{Patient consent for publication}

Not applicable.

\section{Competing interests}

The authors declare that they have no competing interests.

\section{References}

1. Siegel RL, Miller KD and Jemal A: Cancer statistics, 2016. CA Cancer J Clin 66: 7-30, 2016.

2. Cheng Y, Jin Z, Agarwal R, Ma K, Yang J, Ibrahim S, Olaru AV, David S, Ashktorab H, Smoot DT, et al: LARP7 is a potential tumor suppressor gene in gastric cancer. Lab Invest 92: 1013-1019, 2012.

3. Wang J, Guo W, Wu Q, Zhang R and Fang J: Impact of combination epidural and general anesthesia on the long-term survival of gastric cancer patients: A retrospective study. Med Sci Monit 22: 2379-2385, 2016.

4. Yamamura S, Imai-Sumida $M$, Tanaka $Y$ and Dahiya R: Interaction and cross-talk between non-coding RNAs. Cell Mol Life Sci 75: 467-484, 2018.

5. Sana J, Faltejskova P, Svoboda M and Slaby O: Novel classes of non-coding RNAs and cancer. J Transl Med 10: 103, 2012.

6. Ponting CP, Oliver PL and Reik W: Evolution and functions of long noncoding RNAs. Cell 136: 629-641, 2009.

7. Pan W, Liu L, Wei J, Ge Y, Zhang J, Chen H, Zhou L, Yuan Q, Zhou $\mathrm{C}$ and Yang M: A functional lncRNA HOTAIR genetic variant contributes to gastric cancer susceptibility. Mol Carcinog 55: 90-96, 2016

8. Huang C, Cao L, Qiu L, Dai X, Ma L, Zhou Y, Li H, Gao M, $\mathrm{Li} \mathrm{W}$, Zhang Q, et al: Upregulation of $\mathrm{H} 19$ promotes invasion and induces epithelial-to-mesenchymal transition in esophageal cancer. Oncol Lett 10: 291-296, 2015.

9. Wang F, Xie C, Zhao W, Deng Z, Yang H and Fang Q: Long non-coding RNA CARLo-5 expression is associated with disease progression and predicts outcome in hepatocellular carcinoma patients. Clin Exp Med 17: 33-43, 2017. 
10. Wang Y, Liu X,Zhang H, Sun L, Zhou Y, Jin H, Zhang H, Zhang H, Liu J, Guo H, et al: Hypoxia-inducible lncRNA-AK058003 promotes gastric cancer metastasis by targeting gamma-synuclein. Neoplasia 16: 1094-1106, 2014.

11. Li L, Zhang L, Zhang Y and Zhou F: Increased expression of LncRNA BANCR is associated with clinical progression and poor prognosis in gastric cancer. Biomed Pharmacother 72: 109-112, 2015.

12. Gong Z, Zhang S, Zeng Z, Wu H, Yang Q, Xiong F, Shi L, Yang J, Zhang W, Zhou Y, et al: LOC401317, a p53-regulated long non-coding RNA, inhibits cell proliferation and induces apoptosis in the nasopharyngeal carcinoma cell line HNE2. PLoS One 9: e110674, 2014.

13. Svoboda M, Slyskova J, Schneiderova M, Makovicky P, Bielik L, Levy M, Lipska L, Hemmelova B, Kala Z, Protivankova M, et al: HOTAIR long non-coding RNA is a negative prognostic factor not only in primary tumors, but also in the blood of colorectal cancer patients. Carcinogenesis 35: 1510-1515, 2014.

14. Salmena L, Poliseno L, Tay Y, Kats L and Pandolfi PP: A ceRNA hypothesis: The rosetta stone of a hidden RNA language? Cell 146: 353-358, 2011.

15. Tay Y, Rinn J and Pandolfi PP: The multilayered complexity of ceRNA crosstalk and competition. Nature 505: 344-352, 2014.

16. Bassett AR, Azzam G, Wheatley L, Tibbit C, Rajakumar T, McGowan S, Stanger N, Ewels PA, Taylor S, Ponting CP, et al: Understanding functional miRNA-target interactions in vivo by site-specific genome engineering. Nat Commun 5: 4640, 2014.

17. Wang J, Liu X, Wu H, Ni P, Gu Z, Qiao Y, Chen N, Sun F and Fan Q: CREB up-regulates long non-coding RNA, HULC expression through interaction with microRNA-372 in liver cancer. Nucleic Acids Res 38: 5366-5383, 2010.

18. Chen X, Chen Z, Yu S, Nie F, Yan S, Ma P, Chen Q, Wei C, $\mathrm{Fu} \mathrm{H}, \mathrm{Xu} \mathrm{T}$, et al: Long noncoding RNA LINC01234 functions as a competing endogenous RNA to regulate CBFB expression by sponging miR-204-5p in gastric cancer. Clin Cancer Res 24 2002-2014, 2018

19. Gu W, Gao T, Sun Y, Zheng X, Wang J, Ma J, Hu X, Li J and Hu M: LncRNA expression profile reveals the potential role of lncRNAs in gastric carcinogenesis. Cancer Biomark 15: 249-258, 2015.

20. Ye H, Liu K and Qian K: Overexpression of long noncoding RNA HOTTIP promotes tumor invasion and predicts poor prognosis in gastric cancer. OncoTargets Ther 9: 2081-2088, 2016.

21. Li F, Huang C, Li Q and Wu X: Construction and comprehensive analysis for dysregulated long non-coding RNA (lncRNA)-associated competing endogenous RNA (ceRNA) network in gastric cancer. Med Sci Monit 24: 37-49, 2018.

22. Robinson MD, McCarthy DJ and Smyth GK: edgeR: A Bioconductor package for differential expression analysis of digital gene expression data. Bioinformatics 26: 139-140, 2010

23. Wickham H (ed): ggplot2: Elegant Graphics for Data Analysis. 1st edition. Springer-Verlag, New York, NY, 2009.

24. Jeggari A, Marks DS and Larsson E: miRcode: A map of putative microRNA target sites in the long non-coding transcriptome. Bioinformatics 28: 2062-2063, 2012.

25. Shannon P, Markiel A, Ozier O, Baliga NS, Wang JT, Ramage D, Amin N, Schwikowski B and Ideker T: Cytoscape: A software environment for integrated models of biomolecular interaction networks. Genome Res 13: 2498-2504, 2003.

26. Livak KJ and Schmittgen TD: Analysis of relative gene expression data using real-time quantitative PCR and the $2^{-\Delta \Delta C}$ method. Methods 25: 402-408, 2001.

27. Therneau T: A Package for Survival Analysis in S. version 2.38, 2015. https://CRAN.R-project.org/package=survival.

28. Muers M: RNA: Genome-wide views of long non-coding RNAs. Nat Rev Genet 12: 742, 2011

29. Kornienko AE, Guenzl PM, Barlow DP and Pauler FM: Gene regulation by the act of long non-coding RNA transcription. BMC Biol 11: 59, 2013.

30. Wahlestedt $C$ : Targeting long non-coding RNA to therapeutically upregulate gene expression. Nat Rev Drug Discov 12: 433-446, 2013.

31. Song H, Sun W, Ye G, Ding X, Liu Z, Zhang S, Xia T, Xiao B $\mathrm{Xi}$ Y and Guo J: Long non-coding RNA expression profile in human gastric cancer and its clinical significances. J Transl Med 11:225, 2013.

32. Chen X: Predicting lncRNA-disease associations and constructing lncRNA functional similarity network based on the information of miRNA. Sci Rep 5: 13186, 2015.

33. Kong R, Zhang EB, Yin DD, You LH, Xu TP, Chen WM, Xia R, Wan L, Sun M, Wang ZX, et al: Long noncoding RNA PVT1 indicates a poor prognosis of gastric cancer and promotes cell proliferation through epigenetically regulating $\mathrm{p} 15$ and $\mathrm{p} 16 . \mathrm{Mol}$ Cancer 14: 82, 2015.
34. Fu H, Wang C, Yang D, Wei Z, Xu J, Hu Z, Zhang Y, Wang W, Yan $\mathrm{R}$ and Cai Q: Curcumin regulates proliferation, autophagy, and apoptosis in gastric cancer cells by affecting PI3K and P53 signaling. J Cell Physiol 233: 4634-4642, 2018.

35. Qiu YS, Liao GJ and Jiang NN: REG3A overexpression suppresses gastric cancer cell invasion, proliferation and promotes apoptosis through PI3K/Akt signaling pathway. Int J Mol Med 41: 3167-3174, 2018

36. Zhang J, Wei W, Jin HC, Ying RC, Zhu AK and Zhang FJ: Programmed cell death 2 protein induces gastric cancer cell growth arrest at the early $\mathrm{S}$ phase of the cell cycle and apoptosis in a p53-dependent manner. Oncol Rep 33: 103-110, 2015.

37. Huang Y, Zhang J, Hou L, Wang G, Liu H, Zhang R, Chen X and Zhu J: LncRNA AK023391 promotes tumorigenesis and invasion of gastric cancer through activation of the PI3K/Akt signaling pathway. J Exp Clin Cancer Res 36: 194, 2017.

38. Wei GH and Wang X: lncRNA MEG3 inhibit proliferation and metastasis of gastric cancer via p53 signaling pathway. Eur Rev Med Pharmacol Sci 21: 3850-3856, 2017.

39. Ooi A, Oyama T, Nakamura R, Tajiri R, Ikeda H, Fushida $S$ and Dobashi Y: Gene amplification of $C C N E 1, C C N D 1$, and $C D K 6$ in gastric cancers detected by multiplex ligation-dependent probe amplification and fluorescence in situ hybridization. Hum Pathol 61: 58-67, 2017.

40. Ju H, Lim B, Kim M, Noh SM, Kim WH, Ihm C, Choi BY, Kim YS and Kang C: SERPINE1 intron polymorphisms affecting gene expression are associated with diffuse-type gastric cancer susceptibility. Cancer 116: 4248-4255, 2010.

41. Li J, Ding Y and Li A: Identification of COL1A1 and COL1A2 as candidate prognostic factors in gastric cancer. World J Surg Oncol 14: 297, 2016.

42. Li CY, Liang GY, Yao WZ, Sui J, Shen X, Zhang YQ, Peng H, Hong WW, Ye YC, Zhang ZY, et al: Integrated analysis of long non-coding RNA competing interactions reveals the potential role in progression of human gastric cancer. Int J Oncol 48: 1965-1976, 2016.

43. Jin B, Jin $\mathrm{H}, \mathrm{Wu} \mathrm{HB}, \mathrm{Xu} \mathrm{JJ}$ and $\mathrm{Li} \mathrm{B}$ : Long non-coding RNA SNHG15 promotes CDK14 expression via miR-486 to accelerate non-small cell lung cancer cells progression and metastasis. J Cell Physiol 233: 7164-7172, 2018.

44. Yu X,Zhao J and He Y: Long non-coding RNA PVT1 functions as an oncogene in human colon cancer through miR-30d-5p/RUNX2 axis. J BUON 23: 48-54, 2018.

45. Gu L, Lu LS, Zhou DL and Liu ZC: UCA1 promotes cell proliferation and invasion of gastric cancer by targeting CREB1 sponging to miR-590-3p. Cancer Med 7: 1253-1263, 2018.

46. Zhao R, Zhang Y, Zhang X, Yang Y, Zheng X, Li X, Liu Y and Zhang Y: Exosomal long noncoding RNA HOTTIP as potential novel diagnostic and prognostic biomarker test for gastric cancer. Mol Cancer 17: 68, 2018.

47. Xue M, Chen LY, Wang WJ, Su TT, Shi LH, Wang L, Zhang W, Si JM, Wang LJ and Chen SJ: HOTAIR induces the ubiquitination of Runx 3 by interacting with Mex $3 b$ and enhances the invasion of gastric cancer cells. Gastric Cancer 21: 756-764, 2018.

48. Yan J, Zhang Y, She Q, Li X, Peng L, Wang X, Liu S, Shen X, Zhang W, Dong Y, et al: Long noncoding RNA H19/miR-675 axis promotes gastric cancer via FADD/caspase 8/caspase 3 signaling pathway. Cell Physiol Biochem 42: 2364-2376, 2017.

49. Pei YF, Zhang YJ, Lei Y, Wu DW, Ma TH and Liu XQ Hypermethylation of the CHRDL1 promoter induces proliferation and metastasis by activating Akt and Erk in gastric cancer. Oncotarget 8: 23155-23166, 2017.

50. Liu H, Zhang M, Xu S, Zhang J, Zou J, Yang C, Zhang Y, Gong C, Kai Y and Li Y: HOXC8 promotes proliferation and migration through transcriptional up-regulation of TGF $\beta 1$ in non-small cell lung cancer. Oncogenesis 7: 1, 2018.

51. Chen D, Maruschke M, Hakenberg O, Zimmermann W, Stief CG and Buchner A: TOP2A, HELLS, ATAD2, and TET3 are novel prognostic markers in renal cell carcinoma. Urology 102: 265 e261-265 e267, 2017.

52. Dong Y, Li J, Han F, Chen H, Zhao X, Qin Q, Shi R and Liu J: High IGF2 expression is associated with poor clinical outcome in human ovarian cancer. Oncol Rep 34: 936-942, 2015.

53. Li Q, Dai Y, Wang F and Hou S: Differentially expressed long non-coding RNAs and the prognostic potential in colorectal cancer. Neoplasma 63: 977-983, 2016.

54. Yao J, Zhou B, Zhang J, Geng P, Liu K, Zhu Y and Zhu W: A new tumor suppressor LncRNA ADAMTS9-AS2 is regulated by DNMT1 and inhibits migration of glioma cells. Tumour Biol 35: 7935-7944, 2014. 